\section{Dental prosthetic rehabilitation of Papillon-Lefèvre syndrome: a case report}

\author{
Shweta M. Patil, ${ }^{1}$ Suryakant B. \\ Metkari, ${ }^{2}$ Shilpa Shetty, ${ }^{3}$ Savita \\ Thakkannavar, ${ }^{2}$ Sachin C. Sarode, ${ }^{4}$ \\ Gargi S. Sarode, ${ }^{4}$ Namrata Sengupta, ${ }^{4}$ \\ Shankargouda Patil ${ }^{5}$ \\ ${ }^{1}$ Department of Prosthodontics, \\ Tatyasaheb Kore Dental College and \\ Research Center, Kolhapur, \\ Maharashtra, India; ${ }^{2}$ Department of \\ Oral Pathology and Microbiology, \\ Tatyasaheb Kore Dental College and \\ Research Center, Kolhapur, \\ Maharashtra, India; ${ }^{3}$ Department of \\ Conservative Dentistry, Tatyasaheb \\ Kore Dental College and Research \\ Center, Kolhapur, Maharashtra, India; \\ ${ }^{4}$ Department of Oral Pathology and \\ Microbiology, Dr. D.Y. Patil Dental \\ College and Hospital, Dr. D.Y. Patil \\ Vidyapeeth, Pimpri, Pune, India; \\ ${ }^{5}$ Department of Maxillofacial Surgery \\ and Diagnostic Sciences, Division of \\ Oral Pathology, College of Dentistry, \\ Jazan University, Jazan, Saudi Arabia
}

\begin{abstract}
Papillon-Lefèvre syndrome (PLS) is a rare disorder characterized by palmar plantar hyperkeratosis and rapidly progressive periodontitis with loss of deciduous and permanent dentition at an early age. It is reported to occur in 1 to 4 individuals per million people. This case report presents highlighting features of the rare PLS in a 17-year old male who complained of discomfort while mastication and it was accompanied with symmetrical, sharply demarcated erythematous plaques involving the skin of the palms and soles, which extended to the finger joints, elbows, and knees. Along with the rough and dry skin, hyperhidrosis of lesion with a foul odor and transverse grooving of nails were noted. The past dental history revealed normal eruption of deciduous teeth followed by pre-mature mobility and shedding in 4-6 months. Permanent teeth also showed normal eruption and early shedding in the next 4-5 years. Complete edentulous maxillary and mandibular arches led to a decrease in alveolar bone and facial heights. There was no similar disorder in the patient's family but family history revealed the consanguineous marriage of the patient's parents.
\end{abstract}

\section{Introduction}

Papillon-Lefèvre syndrome (PLS) is a rare autosomal recessive disorder characterized by palmar plantar hyperkeratosis and rapidly progressive periodontitis, leading to early loss of deciduous and permanent dentition. ${ }^{1}$ The prevalence of this disorder is estimated at approximately 1 to 4 per million in the general population with a carrier rate of 2 to 4 per 1000 population. $^{2}$ The consanguineous marriage of parents has been found in about $20 \%$ to $40 \%$ of the cases.

The clinical manifestations of the PLS start at the ages of 1 and 4 years and there is no racial or gender predominance. Cutaneous and intra-oral lesions are the striking features of this rare disorder. The changes in the skin begin to appear in the initial four years of life, which consist of keratotic lesions on the palms and soles. ${ }^{4}$ The skin of the palms and soles show characteristic thickenings due to hyperkeratosis and usually is accompanied by foulsmelling. The periodontium is also affected early years in the form of loosening of teeth and severe periodontitis. This leads to premature loss of deciduous teeth and eventually the permanent teeth as well. ${ }^{4}$

The causative factors of this disorder are still not clear but genetics and immunodeficiency are considered to be the prominent ones. In recent investigations, mutation of the cathepsin $\mathrm{C}$ gene has been held responsible for the initiation and progression of the disorder. ${ }^{5}$ Due to this mutation, the body of a PLS patient fails to evoke immune responses. The resident pathogens enjoy an unobstructed opportunity to grow and flourish in the body especially in the periodontium. Dentists and dermatologists are at the forefront to notice such intra-oral and cutaneous lesions and should bring them to focus to enlighten the medical literature of PLS.

PLS being a rare disorder the knowledge about this syndrome is very limited among researchers. Thus, this report aims at discussing the features of PLS and its management strategies to give an insight to the clinicians and enhance their understanding of this rare disease.

\section{Case Report}

A 17-year old male patient reported with complaining of discomfort while chewing of food due to loss of teeth for 7-8 years. The past dental history revealed that his primary teeth had erupted in a normal manner but teeth became mobile and started shedding over 4-6 months. There had also
Correspondence: Sachin C. Sarode, Department of Oral Pathology and Microbiology, Dr. D.Y. Patil Dental College and Hospital, Sant-Tukaram Nagar, Pimpri, Pune, 411018 India.

Tel.: +91.9922491465.

E-mail: drsachinsarode@gmail.com

Key words: Palmar planter hyperkeratosis; Papillon-Lefèvre syndrome; periodontitis; complete denture.

Conflicts of interests: the authors declare no conflicts of interests.

Ethics approval and consent to participate: The present paper is a case report and hence ethical approval is not mandatory for its publication.

Informed consent: As per the protocol of our Institution, informed consent for use of clinical and biological material for publication is taken from each and every patient visiting to Out Patient Department.

Received for publication: 23 July 2020.

Revision received: 24 August 2020.

Accepted for publication: 7 September 2020.

This work is licensed under a Creative Commons Attribution NonCommercial 4.0 License (CC BY-NC 4.0).

(C) Copyright: the Author(s), 2020

Licensee PAGEPress, Italy

Clinics and Practice 2020; 10:1285

doi:10.4081/cp.2020.1285

been a normal eruption of all permanent teeth but again tooth mobility occurred with the shedding of all permanent teeth in the period of next 4-5 years. Family history revealed the consanguineous marriage of his parents. There was no similar disorder in the family.

Physical examination revealed there were symmetrical, well-defined erythematous keratotic confluent plaques affecting the skin of the palms and soles, which extended to the dorsal surface of the hand and feet (Figure 1). The skin was rough and dry on palpation. Skin appeared dirty colored with painful fissures. Hyperhidrosis of lesion was noted which resulted in foul odor. There was transverse grooving of nails. The hair appeared normal.

Intraoral examination revealed complete edentulous maxillary and mandibular arches (Figure 2). The mucosa of the edentulous area appeared normal. Early loss of teeth and loss of alveolar bone had resulted in decreased facial height. All laboratory investigations were within normal limits.

Considering overall findings maxillary and mandibular complete denture were 
planned. Classical steps of denture fabrication were followed. Casts were prepared based on the primary impressions with irreversible hydrocolloid material and subsequently, special trays were fabricated. After obtaining final impressions with light body polyvinyl siloxane impression material, jaw relation record procedure and the casts were mounted on the semi-adjustable articulator (Hanau Wide-Vue ${ }^{\mathrm{TM}}$ ). After making of teeth arrangement, denture try-in was completed and dentures were fabricated in high impact heat polymerized acrylic resin (Figure 2). Usual recall visits were followed up and necessary adjustments were done. The patient was informed regarding oral rehabilitation with implant prosthesis at a later stage.

\section{Discussion}

Papillon-Lefèvre syndrome is a very rare, inherited disease, which shows premature loss of deciduous, and permanent teeth. Due to underlying pathogenesis, the eruption of deciduous dentition is associated with severe gingivitis and subsequent rapid destruction of periodontium. The resulting periodontitis is unresponsive to traditional periodontal treatment and hence primary dentition is exfoliated prematurely by the age of 4 to 5 years. After exfoliation of deciduous teeth gingiva appears healthy due to the elimination of potential for the microbial colonization. The same sequence of events recurs as permanent dentition erupts, leading to early exfoliation and without treatment, most of the permanent teeth may fall at an early age. The complete edentulous arches in the present case represent the perfect disease progression model associated with PLS.

Cutaneous changes include sharply demarcated erythematous keratotic lesions on the palms, soles, dorsum of the hand and feet. ${ }^{6}$ In agreement with the findings in the literature, hyperkeratotic cutaneous lesions, hyperhidrosis, and pyogenic infections were observable features in the present case. Other features that have been reported consist of intracranial calcification and choroid plexus and retardation of somatic development, hyperhidrosis, spider fingers and retarded mental health. ${ }^{7}$ In addition to this, the patient may also show increased susceptibility to bacterial infection leading to pyogenic infection of the skin and even otitis media due to the dysfunction of lymphocytes and leukocytes. ${ }^{8}$

Multiple etiological factors are associated with the syndrome like immunological alteration, genetic mutations and the role of bacteria. Impairment of neutrophil chemo- taxis, phagocytosis and bactericidal activity has been responsible for the initiation and proliferation of the disorder. ${ }^{2}$ The normal physiological functions of neutrophils are affected. There is a serine protease deficiency in the neutrophils, which reduces their chemotactic capability. ${ }^{9}$ Thus, the body faces a dysregulated immune response and instead of phagocytosing the pathogens, it allows their overgrowth. The neutrophils also release proinflammatory cytokines higher than normal, thus, generating a vicious cycle of chronic inflammation in the periodontium. Virulent gram-negative anaerobic pathogens (Actinobacillus actinomycetemcomitans) causing periodontal damage along with alterations in the polymorphonuclear leukocyte functions ${ }^{10}$ and a defect of immune-mediated mechanisms including reduced lymphocyte to pathogens, depression of helper/depressor $\mathrm{T}$ cell ratio, deficient monocyte function, the elevation of serum IgG and degenerative changes of plasma cells ${ }^{11}$ have been thought to be the pathogenic mechanisms.

Recently, it has been noted that the cathepsin- $\mathrm{C}$ gene is expressed in the epithelial regions such as the palms, soles, knees and gingiva. A mutation of this gene results in PLS. The effect of this mutation is the
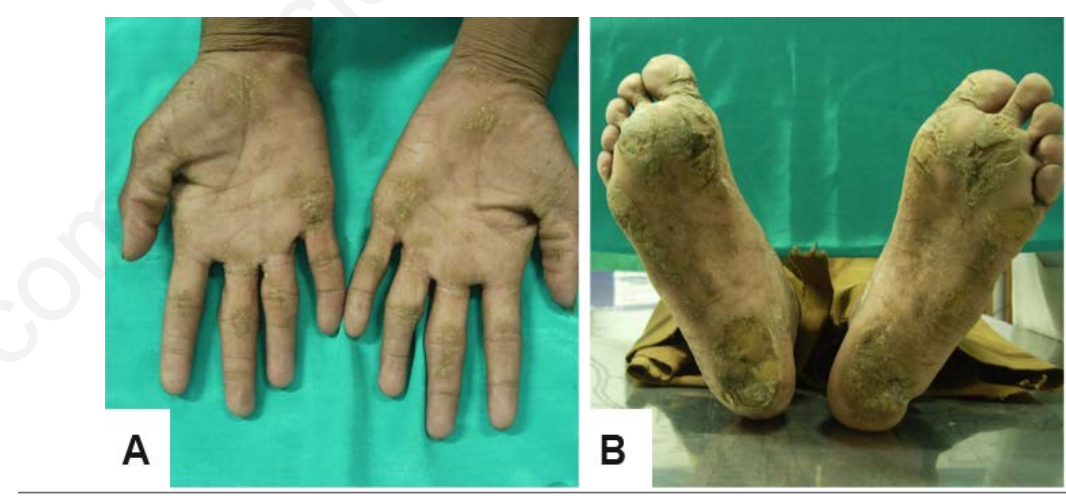

Figure 1. Clinical photograph showing hyperkeratosis affecting palms (A) and soles of feet $(B)$.

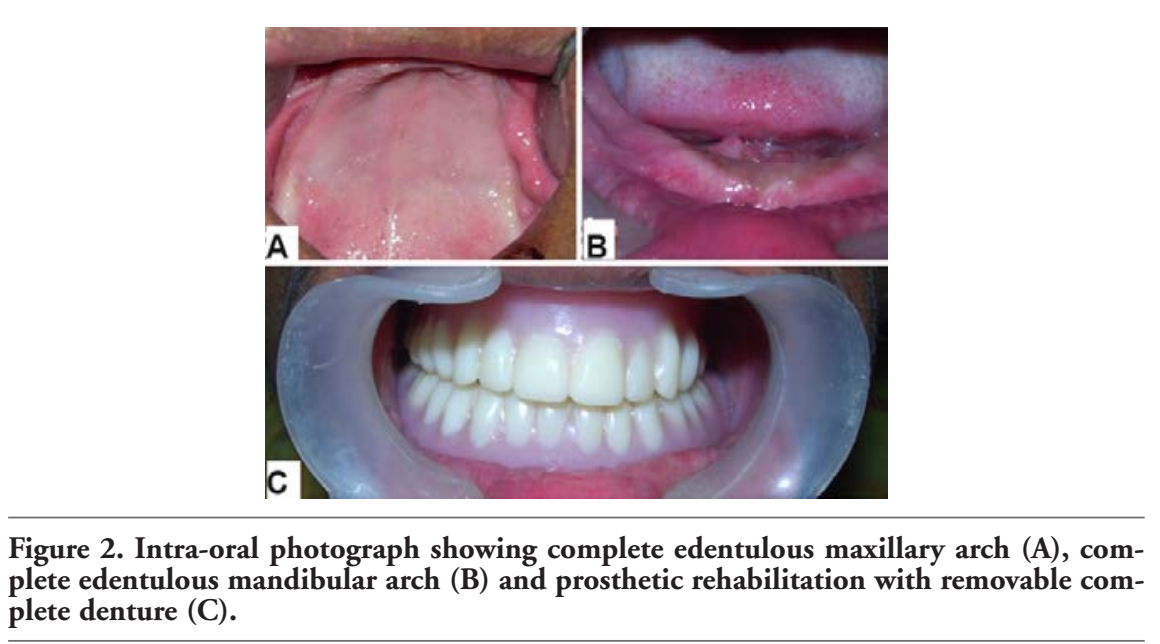

Figure 2. Intra-oral photograph showing complete edentulous maxillary arch (A), complete edentulous mandibular arch (B) and prosthetic rehabilitation with removable complete denture $(C)$.

serine protease deficiency of the neutrophils. The loss of function of serine protease makes the body incapable to get rid of the periodontal pathogens, which has been thought to be the major cause of pre-pubertal periodontitis in this syndrome. Surprisingly, the mutation of this gene also results in two other closely related conditions: the Haim- Munk syndrome and prepubertal periodontitis. The common clinical finding in all these three syndromes is the early onset of severe periodontitis. ${ }^{8}$ A missense type of mutation of the cathepsin-C gene has also been reported quite recently, which has proven to be lethal. ${ }^{12}$

In the present case, the skin and oral findings have a striking resemblance to the descriptions of this syndrome. The skin lesions appeared in the first and second decades of life. These lesions worsened during the winter season, with deepening of fissures and recurrent pyogenic infections. The oral manifestations usually occur simultaneously with the onset of palmar plantar hyperkeratosis. After the eruption of deciduous dentition, rapidly progressing periodontitis set in leading to their premature loss. Oral manifestations also include gingivitis and halitosis with pus exudation from a periodontal pocket. There is a deep 
periodontal pocket formation, bone resorption but no root resorption. Sometimes, root completion might be incomplete and microdontia might be found in a few PLS cases, however it was not reported in the present case. ${ }^{13}$ Primary loss of alveolar bone around the teeth causes loss of teeth resulting in edentulism. Gingiva reverts to normal health after exfoliation of teeth. Similar events happen with the permanent dentition. ${ }^{14}$ Hypermobility and early exfoliation of teeth make mastication a very difficult activity. Fabrication of denture in these patients is difficult because the patients become edentulous at a young age and the alveolar ridges are severely atrophic due to early-onset periodontitis. Moreover, complete edentulous condition resorbs the alveolar bone reducing the vertical dimension of occlusion.

Histopathological sections of the gingival epithelium of a PLS individual usually show excessive keratinization. ${ }^{15}$ Acanthosis, hyperplasia and inflammatory cell infiltrate (neutrophils, lymphocytes and plasma cells) are also evident. Since the diagnosis of PLS is purely based on the clinical manifestations, a biopsy was not carried out in the present case. Radiographically, loss of alveolar bone is quite noticeable around incisors and first molars in the ages of puberty. ${ }^{15}$ Eventually, it affects minimum to a maximum number of teeth and teeth without any supporting structures around them appear to be floating.

The cutaneous and oral lesions of PLS should be differentiated from other diseases, which present similar kind of signs and symptoms. The differential diagnosis of PLS can be a few osteolytic lesions such as pre-pubertal periodontitis, Haim-Munk syndrome, Takahara's syndrome, Greither's syndrome, Keratoderma hereditarium mutilans and leukemia. ${ }^{16}$ In pre-pubertal periodontitis, the sign of palmoplantar keratoderma is usually absent. Some of the conditions like hypophosphatasia, leukemia, acrodynia, histiocytosis, Takahara's syndrome and cyclic neutropenia show the association with periodontal lesions followed by early loss of teeth. But these conditions are not found to be associated with hyperkeratosis of palmoplantar regions. On the other hand, some diseases such as Keratoderma hereditarium mutilans, Greither's syndrome, Howel-Evan's syndrome show cutaneous keratotic lesions but not periodontal pathologies. A severe deficiency of vitamin A can also present hyperkeratotic cutaneous lesions and dura and choroid plexus calcifications. ${ }^{17}$ Thus, PLS should be differentiated from all such lesions and conditions before concluding the final diagnosis.

The management of this uncommon disorder is very challenging. The skin manifestations of PLS are usually treated with emollients. Salicylic acid, topical steroids and urea may be added for enhancement of their effect. Due to the role of retinoids in the differentiation of epithelium, oral retinoids have an important role in the management of PLS. Loss of dentition can be prevented with proper periodontal treatment including scaling, root planning and oral hygiene instructions and $0.2 \%$ chlorhexidine gluconate mouth rinses, along with antibiotic therapy and retinoids but it will be successful if done before the onset of the eruption of permanent teeth. Teeth having deep periodontal pockets are treated by flap surgical procedures. Early extraction of teeth has been carried out to prevent bone loss and then a solid foundation has been laid for successful artificial denture fabrication. ${ }^{18}$

PLS affects an individual socially, physically and psychologically. The premature loss of teeth will also concern the individual aesthetically. Thus, oral rehabilitation is a must. Partial dentures, complete dentures or implant-supported prosthesis are the most recommended treatment modalities for PLS patients. The prosthetic approach is an agespecific treatment involving the fabrication of removable partial or complete dentures initially. At an early age, during mixed dentition period, missing teeth are replaced with a removable prosthesis. Smaller size teeth are selected, giving space between the teeth that resemble deciduous dentition. After insertion of the removable prosthesis, a routine follow-up of the patient has to be carried out. Rehabilitation of an edentulous PLS patient can be done with conventional complete dentures, modified complete dentures, implant-supported prosthesis, overdentures, or a combination of these; however, the outcome of treatment will be based on the complaint, availability of supporting bone, expectations and financial conditions of the patient. ${ }^{15}$

Some clinicians propose the usage of digital technologies in psychologically traumatized PLS patients, wherein, extraction and denture delivery is performed on the very same day. ${ }^{13}$ Computer-supported technologies and machinery make this step possible. Prosthetic rehabilitation provides a psychological boost up to the patient by restoring function and esthetic appearance. It also reliefs the patient's parents from psychological stress. Hence, a multidisciplinary approach is important to treat the patient with PLS

\section{Conclusions}

The present case report displays a classical presentation of PLS along with complete oral rehabilitation using complete denture. Dental surgeons can quite easily diagnose PLS due to classical clinical manifestations and rapidly progressive periodontitis. Parents of the individual, if notice keratotic plaques on the palms or plantar surfaces of the feet of their children, should responsibly consult a dermatologist as well as dental surgeons for early treatment. There are very few cases of PLS reported in the literature due to the rarity of the disease and hence, there is a dire need for sensitization of the general population about PLS syndrome. This case report will benefit the clinicians to widen their scope of knowledge regarding PLS and will enhance their understanding of planning a treatment protocol for a PLS patient.

\section{References}

1. Machado RA, Cuadra-Zelaya FJM, Martelli-Júnior $\mathrm{H}$, et al. Clinical and molecular analysis in Papillon-Lefèvre syndrome. Am J Med Genet A 2019;179:2124-31.

2. Janjua SA, Khachemoune A. PapillonLefèvre syndrome: case report and review of the literature. Dermatol Online J 2004;10:13.

3. Khan FY, Jan SM, Mushtaq M. Papillon-Lefèvre syndrome: Case report and review of the literature. J Indian Soc Periodontol 2012;16:261-5.

4. Silva TS, Lacerda PN, Rêgo FP, Rêgo VR. Papillon-Lefèvre syndrome. An Bras Dermatol 2018;93:771-2.

5. De Haar SF, Jansen DI, Schoenmaker T, et al. Loss-of-function mutations in cathepsin $\mathrm{C}$ in two families with Papillon-Lefèvre syndrome are associated with deficiency of serine proteinases in PMNs. Hum Mutat 2004;23:524.

6. Singla A, Sheikh S, Jindal SK, Brar R. Papillon Lefevre syndrome: Bridge between Dermatologist and Dentist. J Clin Exp Dent 2010;2:43-6.

7. Gorlin RJ, Cohen MM, Levin LS. Syndromes of the Head and Neck, 3rd ed. Oxford: Oxford University Press; 1990. pp. 853-5.

8. Shah J, Goel S. Papillon Lefevre syndrome: two case reports. Indian J Dent Res 2007;18:210-13.

9. Roberts H, White P, Dias I, et al. Characterization of neutrophil function in Papillon-Lefèvre syndrome. J Leukoc Biol 2016;100:433-44. 
10. González JR, Chabrier L, Rodriguez RJ. Papillon-Lefevre syndrome: a case report and review of the literature. P R Health Sci J 1997;16:279-81.

11. Hattab FN, Amin WM. PapillonLefèvre syndrome with albinism: a review of the literature and report of 2 brothers. Oral Surg Oral Med Oral Pathol Oral Radiol Endod 2005;100:709-16.

12. Meenu S, Pradeep B, Ramalingam S, et al. Papillon-Lefèvre syndrome (PLS) with novel compound heterozygous mutation in the exclusion and Peptidase C1A domains of Cathepsin $\mathrm{C}$ gene. Mol
Biol Rep 2020;29:1-7.

13. Giannetti L, Apponi R, Dello Diago $\mathrm{AM}$, et al. Papillon-Lefèvre syndrome: oral aspects and treatment. Dermatol Ther 2020;28:e13336.

14. Abdulwassie H, Dhanrajani PJ, Jiffry A. Papillon Lefevre syndrome. IReappraisal of etiology, clinical features and treatment, II- Oral rehabilitation using osseointegrated implants. Indian J Dent Res 1996;7:63-70.

15. Sreeramulu B, Shyam ND, Ajay P, Suman P. Papillon-Lefèvre syndrome: clinical presentation and management options. Clin Cosmet Investig Dent
2015;7:75-81

16. Singh AP, Chaitra TR, Singh SP, Kulkarni AU. Initial expression of the Papillon-Lefèvre syndrome in consanguine family. Case Rep 2012;2012:bcr2012006313.

17. Jose J, Bartlett K, Salgado C, Gutierrez NM. Papillon-Lefevre syndrome: review of imaging findings and current literature. Foot Ankle Spec 2015;8:13942.

18. Shafer WG, Hine MK, Levy BM. A text book of oral pathology. 4th ed. WB Saunders Company; 1997. 